\title{
Network Probabilistic Connectivity: Using Node Cuts
}

\author{
Denis A. Migov, Olga K. Rodionova, \\ Alexey S. Rodionov ${ }^{1}$, and Hyunseung $\mathrm{Choo}^{2, \star}$ \\ ${ }^{1}$ Institute of Computational Mathematics and Mathematical Geophysics \\ Siberian Division of the Russian Academy of Science \\ Novosibirsk, Russia \\ +383-3326949 \\ alrod@rav.sscc.ru \\ ${ }^{2}$ School of Information and Communication Engineering \\ Sungkyunkwan University \\ 440-746, Suwon, Korea \\ $+82-31-290-7145$ \\ choo@ece.skku.ac.kr
}

\begin{abstract}
The new algorithmic technique which allow higher efficiency in the precise calculation of the reliability of an undirected graph with absolutely reliable nodes and unreliable edges is considered in this paper. As graph's reliability we mean its probabilistic connectivity. The proposed technique is based on the reduction of the dimension of a calculated graph by considering its node's cuts. Comprehensive computer simulation results show the advantages of the proposed algorithms, that the calculation time decreases significantly in comparison with existent methods.
\end{abstract}

\section{Introduction}

The task of calculating or estimating the probability of whether the network is connected (often referred to as its reliability, is the subject of much research due to its significance in a lot of applications, communication networks included. The problem is known to be NP-hard irrelative of whether the unreliable edges or nodes or both are considered. Most explored is the case of absolutely reliable nodes and unreliable edges that corresponds to real networks in which the reliability of nodes is much higher than that of edges. The transport and radio networks are good examples. Usually the estimations of a network reliability are considered. Yet we can conduct the exact calculation of reliability for networks with dimension of a practical interest by taking into consideration some special features of real network structures and based on modern high-speed computers. In [1] the technique was proposed that allows faster calculation based on reduction of simple chains and/or branching by them. This technique, in its turn, is based on the well-known factoring (branching) algorithm (often called MooreShannon algorithm [2]) uses branching on the alternative states for an arbitrary

\footnotetext{
^ Corresponding author.
} 
edge. Some good approaches are proposed in 34] but, as it is shown in [1], it is possible to improve them. In this paper we present new approach to reducing the task dimension by considering 2 -node cuts. The proper theorems are proven in this paper to support the proposed methods.

The rest of the paper is organized as follows: in section 2 the basic notations and definitions are presented. In section 3 we prove the basic theorems while section 4 is devoted to computer algorithm and experimental results. Section 5 is the brief conclusion.

\section{Basic Notations and Definitions}

We denote an undirected graph as $G=(X, U)$, where $X$ is a set of nodes and $U$ - a set of edges of $G$. For each edge $u_{i} \in U$ the probability of its existence $p_{i}$ is given. Further on we refer to this probability as edge reliability.

The simple event is an individual realization of a graph that is obtained by checking existence or non-existence of every edge.

The probability of simple event is obviously equal to

$$
P(E)=\prod_{i \in E x} p_{i} \prod_{i \notin E x}\left(1-p_{i}\right),
$$

where $E x$ is the set of numbers of existent edges.

An arbitrary event (union of some simple events) we will consider as successful if all graphs that are correspondent to these simple events are connected that is that all nodes can be connected by existent edges.

Reliability of graph $G, R_{P}(G)$, is the probability of graph $G$ being connected that is the probability of event that is the union of all successful events and of them only.

Let $\{x, y\}$ be an arbitrary 2-node cut in $G$ which divides it into 2 subgraphs $G_{1}$ and $G_{2}$ as is shown in Fig. 1. Note that for any fixed cut $\{x, y\} G_{1}$ and $G_{2}$ are not defined unambiguously in general case. For example, if there is an edge between $x$ and $y$ then it can belongs as to $G_{1}$ as to $G_{2}$.

We denote graphs that are obtained from $G_{1}$ and $G_{2}$ by contracting nodes $x$ and $y$ as $G_{1}^{\prime}$ and $G_{2}^{\prime}$, correspondingly. Let $z$ be the node that is obtained by contracting nodes $x$ and $y$.

\section{Using Node Cuts for Reducing the Task Dimension}

\subsection{Reliability of Graph That Is Divided into 2 Components by 2-Node Cut}

Here we prove the main theorem that correlates the reliability of a graph $G$ with reliabilities of graphs $G_{1}, G_{1}^{\prime}, G_{2}$, and $G_{2}^{\prime}$. 

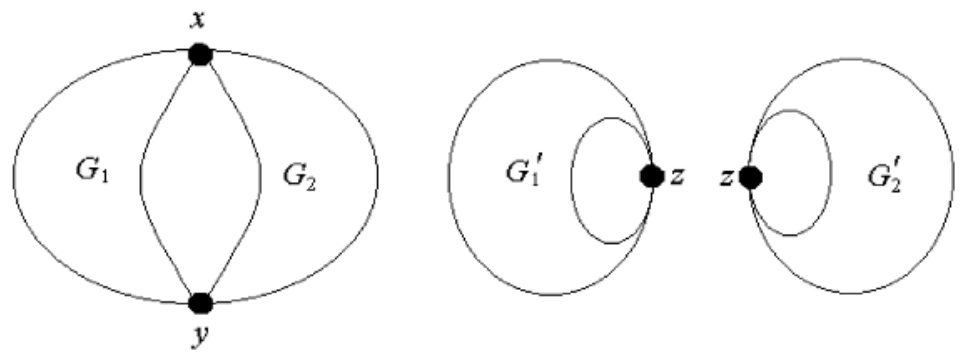

Fig. 1. Components of a graph $G$ and auxiliary graphs

Theorem 1. The following equation is true for any graph $G$ that is divided into 2 components $G_{1}$ and $G_{2}$ by 2-node cut:

$$
\begin{aligned}
R_{P}(G)= & R_{P}\left(G_{1}\right) \cdot\left(R_{P}\left(G_{2}^{\prime}\right)-R_{P}\left(G_{2}\right)\right)+ \\
& R_{P}\left(G_{2}\right) \cdot\left(R_{P}\left(G_{1}^{\prime}\right)-R_{P}\left(G_{1}\right)\right)+R_{P}\left(G_{1}\right) \cdot R_{P}\left(G_{2}\right) .
\end{aligned}
$$

Proof. Let us denote:

$A$ - union of all simple events that correspond to connected $G_{1}$ and $G_{2}^{\prime}$ and disconnected $G_{2}$.

$B$ - union of all simple events that correspond to connected $G_{2}$ and $G_{1}^{\prime}$ and disconnected $G_{1}$.

$C$ - union of all simple events that correspond to connected $G_{1}$ and $G_{2}$.

$S$ - union of all simple events that correspond to connected $G$.

First we need prove the following statements:

1) Events $A, B$, and $C$ are pairwise antithetical, that is $A \cap B=A \cap C=$ $B \cap C=\emptyset$.

2) $A \cup B \cup C=S$.

The truth of the first statement is clear from the definitions of $A, B$, and $C$. Let us prove the second statement.

First we show the inclusion $A \subseteq S$. Let us consider an arbitrary simple event in $A$ such that $G_{1}$ and $G_{2}^{\prime}$ are connected and $G_{2}$ is disconnected.

Let $x_{1}$ and $x_{2}$ be two arbitrary nodes of $G_{2}$. $G_{2}^{\prime}$ is connected so there is at least one path from $x_{1}$ to $z$ and at least one path from $x_{2}$ to $z$ in $G_{2}^{\prime}$. Thus there are paths from $x_{1}$ to $x$ or $y$ and from $x_{2}$ to $x$ or $y$ in $G_{2}$. And if there exist paths from $x_{1}$ to $x$ and from $x_{2}$ to $x$ or from $x_{1}$ to $y$ and from $x_{2}$ to $y$, then, obviously, $x_{1}$ and $x_{2}$ are connected by a path in $G_{2}$.

Now we will discuss the case when there are paths from $x_{1}$ and $x_{2}$ to different nodes $(x$ or $y)$. Without loss of generality we can assume that there are paths from $x_{1}$ to $x$ and from $x_{2}$ to $y . G_{1}$ is connected so there exists a path from $x$ to $y$ in $G_{1}$ and, consequently, there exists a path from $x_{1}$ to $x_{2}$ through nodes $x$ and $y$ (see Fig!2 a)). 


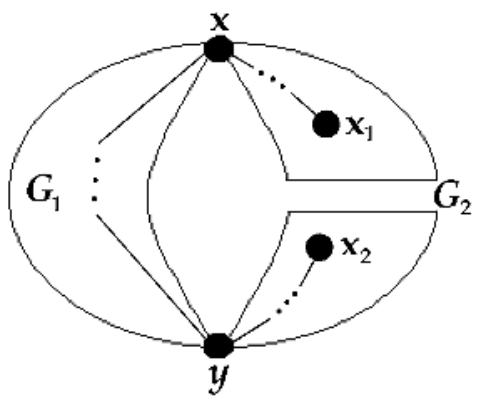

a)

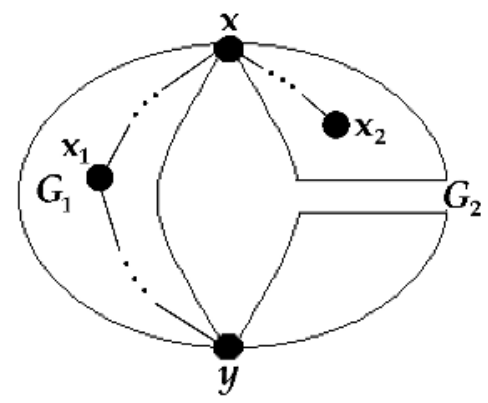

b)

Fig. 2. Case of connected $G_{1}$ and $G_{2}^{\prime}$ and disconnected $G_{2}$

If $x_{1}$ is in $G_{1}$ and $x_{2}$ is in $G_{2}$ then $x_{1}$ is connected to both $x$ and $y$ and $x_{2}$ is connected to $x$ or to $y$ but not to both. Let ut assume that $x_{2}$ is connected to $x$ for certainty. Thus $x_{1}$ and $x_{2}$ are connected by a path in $G$ (see Fig $2 \mathrm{~b}$ )).

Thus any arbitrary pair of nodes in $G$ are connected by at least one path in the case of event $A$ and so $A \subseteq S$.

The inclusion $B \subseteq S$ is proved similar to $A \subseteq S$.

The inclusion $C \subseteq S$ is clear because a connected graph corresponds to any simple event in $C$. Thus $A \cup B \cup C \subseteq S$.

Now we transfer to proving the reverse inclusion, that is $A \cup B \cup C \supseteq S$.

Let us consider an arbitrary simple event $E$ in $S$, that is some partial realization of $G$ that is connected.

We need in three additional events:

$D_{1}$ — union of all simple events that corresponds to connected $G_{1}$ and disconnected $G_{2}^{\prime}$.

$D_{2}$ - union of all simple events that corresponds to connected $G_{2}$ and disconnected $G_{1}^{\prime}$.

$D$ - union of all simple events that corresponds to disconnected $G_{1}$ and disconnected $G_{2}$.

It is clear that $A \cup B \cup C \cup D_{1} \cup D_{2} \cup D=\Omega$ where $\Omega$ is the complete event space. Note that events $D_{1}, D_{2}$ and $D$ are obviously pairwise antithetical.

Let $E \notin A \cup B \cup C$. Then, according to the statement above, $E \in D_{1} \cup D_{2} \cup D$. Now we consider the cases $E \in D_{1}, E \in D_{2}$, and $E \in D$ separately.

1. $E \in D$. In this case $G_{1}$ and $G_{2}$ are disconnected so $G$ is disconnected also. We have the contradiction because $E$ is a connected realization of $G$.

2. $E \in D_{1}$. Let $x_{1}$ and $x_{2}$ be a pair of disconnected nodes in $G_{2}^{\prime}$ (such pair exists according to the definition of $D_{1}$ ). Specifically there is no path from $x_{1}$ to $x_{2}$ through $z$ and this node corresponds to a path through connected $G_{1}$. Consequently they are disconnected in $G$. We have contradiction again.

3. $E \in D_{2}$. The contradiction is obtained similar to the case above. 
Thus $E \notin D_{1} \cup D_{2} \cup D$ so $E \in A \cup B \cup C$ and, consequently, $S \subseteq A \cup B \cup C$. Then from $A \cup B \cup C \subseteq S$ we obtain $A \cup B \cup C=S$.

Now according to statements 1) and 2) we obtain $R(G)=P(S)=P(A)+$ $P(B)+P(C)$.

The event $C$ we can represent as intersection of two events which corresponds to connectivity of $G_{1}$ and connectivity of $G_{2}$, correspondingly. These events are obviously independent. Thus $P(C)=R\left(G_{1}\right) \cdot R\left(G_{2}\right)$.

The event $A=A_{1} \cap A_{2}$, where $A_{1}$ corresponds to connectivity of $G_{1}$ and $A_{2}$ corresponds to connectivity of $G_{2}^{\prime}$ and non-connectivity of $G_{2}$. These events are also independent so $P(A)=P\left(A_{1}\right) \cdot P\left(A_{2}\right)$.

Ii is clear that $P\left(A_{1}\right)=R\left(G_{1}\right)$. For obtaining $P\left(A_{2}\right)$ we present $A_{2}$ as intersection of events $A_{21}$ and $A_{22}$, where $A_{21}$ corresponds to non-connectivity of $G_{2}$ and $A_{22}$ corresponds to connectivity of $G_{2}^{\prime}$. Events $A_{21}$ and $A_{22}$ are dependent ones so $P\left(A_{2}\right) \neq P\left(A_{21}\right) \cdot P\left(A_{22}\right)$. Let us derive the probability of $A_{2}$ in the following way.

By definition of $A_{21}$ the event $\bar{A}_{21}$ corresponds to connectivity of $G_{2}$ thus $\bar{A}_{21} \subseteq A_{22}$ and $P\left(\bar{A}_{21}\right)=R\left(G_{2}\right)$. We have the obvious equivalence: $A_{22} \cap A_{21}=$ $A_{22} \backslash \bar{A}_{21}$. From this we obtain

$$
P\left(A_{2}\right)=P\left(A_{21} \cap A_{22}\right)=P\left(A_{22} \backslash \bar{A}_{21}\right) .
$$

From $\bar{A}_{21} \subseteq A_{22}$, we have

$$
P\left(A_{2}\right)=P\left(A_{22} \backslash \bar{A}_{21}\right)=P\left(A_{22}\right)-P\left(\bar{A}_{21}\right)=R\left(G_{2}^{\prime}\right)-R\left(G_{2}\right) .
$$

So

$$
P(A)=P\left(A_{1}\right) \cdot P\left(A_{2}\right)=R\left(G_{1}\right) \cdot\left(R\left(G_{2}^{\prime}\right)-R\left(G_{2}\right)\right) .
$$

In similar way we obtain that

$$
P(B)=R\left(G_{2}\right) \cdot\left(R\left(G_{1}^{\prime}\right)-R\left(G_{1}\right)\right) .
$$

As a result we have

$$
\begin{aligned}
R(G)= & P(A)+P(B)+P(C)=R\left(G_{1}\right) \cdot\left(R\left(G_{2}^{\prime}\right)-R\left(G_{2}\right)\right)+ \\
& R\left(G_{2}\right) \cdot\left(R\left(G_{1}^{\prime}\right)-R\left(G_{1}\right)\right)+R\left(G_{1}\right) \cdot R\left(G_{2}\right),
\end{aligned}
$$

just as expected.

\subsection{Reliability of a Graph That Is Divided into $k$ Components by 2-Node Cut}

Now let us consider the case when 2-node cut divides graph $G$ into $k$ subgraphs $G_{1}, G_{2}, \ldots, G_{k}, k>2$.

First we need define a intermediate function $S_{k}^{i}$. Let us have $2 k$ numbers $p_{1}, \ldots, p_{k}, p_{1}^{\prime}, \ldots, p_{k}^{\prime}$. Then $S_{k}^{i}$ is equal to a sum of all possible productions of these numbers that satisfy the following restrictions: 
1) each item has $k$ co-factors;

2) each item has no co-factors with equal indexes;

3 ) each item has exactly $i$ co-factors with stroke.

Thus

$$
\begin{aligned}
& S_{k}^{0}=p_{1} p_{2} p_{3} \ldots p_{k}, \\
& S_{k}^{1}=p_{1}^{\prime} p_{2} p_{3} \ldots p_{k}+p_{1} p_{2}^{\prime} p_{3} \ldots p_{k}+\ldots+p_{1} p_{2} p_{3} \ldots p_{k}^{\prime}, \\
& S_{k}^{2}=p_{1}^{\prime} p_{2}^{\prime} p_{3} \ldots p_{k}+p_{1}^{\prime} p_{2} p_{3}^{\prime} \ldots p_{k}+\ldots+p_{1} p_{2} p_{3} \ldots p_{k-1}^{\prime} p_{k}^{\prime}, \\
& \quad \ldots \\
& \begin{aligned}
S_{k}^{k-1} & =p_{1} p_{2}^{\prime} p_{3}^{\prime} \ldots p_{k}^{\prime}+p_{1}^{\prime} p_{2} p_{3}^{\prime} \ldots p_{k}^{\prime}+\ldots+p_{1}^{\prime} p_{2}^{\prime} p_{3}^{\prime} \ldots p_{k-1}^{\prime} p_{k} .
\end{aligned}
\end{aligned}
$$

As $p_{i}$ and $p_{i}^{\prime}$ we consider reliabilities of $G_{i}$ and $G_{i}^{\prime}$ where $G_{i}^{\prime}$ is obtained from $G_{i}$ by contracting nodes $x$ and $y$.

Theorem 2. The following equation is true:

$$
R(G)=\sum_{i=0}^{k-1}(-1)^{k-i+1} S_{k}^{i}
$$

Proof. We prove the theorem by induction.

1. For $k=2$ we obtain the equation (2) that is proved to be true in previous consideration.

2. Let (9) be true for some $k$. Let us prove it for $k+1$.

Let $p_{0}$ be the reliability of $G_{1} \cup \ldots \cup G_{k}$ and $p_{k+1}$ be the reliability of $G_{k+1}$. From (2)) we have

$$
\begin{aligned}
R(G)= & p_{k+1}\left(p_{0}^{\prime}-p_{0}\right)+p_{0}\left(p_{k+1}^{\prime}-p_{k+1}\right)+p_{0} p_{k+1}= \\
& p_{k+1} p_{0}^{\prime}+p_{k+1}^{\prime} p_{0}-p_{0} p_{k+1} .
\end{aligned}
$$

But, by the assumption of induction

$$
p_{0}=\sum_{i=0}^{k-1}(-1)^{k-i+1} S_{k}^{i},
$$

and, obviously, $p_{0}^{\prime}=p_{1}^{\prime} \ldots p_{k}^{\prime}$. Thus

$$
R(G)=p_{1}^{\prime} \ldots p_{k}^{\prime} p_{k+1}+p_{0}\left(p_{k+1}^{\prime}-p_{k+1}\right) .
$$

By simple substitution we can obtain the following property of $S_{k}^{i}$ :

$$
S_{k+1}^{i}=S_{k}^{i-1} p_{k+1}^{\prime}+S_{k}^{i} p_{k+1}, \quad 0<i<k .
$$

For $i=0$ we have $S_{k+1}^{0}=S_{k}^{0} p_{k+1}$ and for $i=1 S_{k+1}^{k}=S_{k}^{k-1} p_{k+1}^{\prime}+p_{1}^{\prime} p_{2}^{\prime} \ldots p_{k}^{\prime} p_{k+1}$. 
Thus for odd $k$ we have

$$
\begin{aligned}
& \sum_{i=0}^{k}(-1)^{k-i+2} S_{k+1}^{i}=-S_{k+1}^{0}+S_{k+1}^{1}-\ldots-S_{k+1}^{k-1}+S_{k+1}^{k}= \\
& -S_{k}^{0} p_{k+1}+S_{k}^{0} p_{k+1}^{\prime}+S_{k}^{1} p+\ldots-S_{k}^{k-1} p_{k+1}+S_{k}^{k-1} p_{k+1}^{\prime}+p_{1}^{\prime} p_{2}^{\prime} \ldots p_{k}^{\prime} p_{k+1}= \\
& \left(p_{k+1}^{\prime}-p_{k+1}\right)\left(S_{k}^{0}-S_{k}^{1}+S_{k}^{2}-\ldots+S_{k}^{k-1}\right)+p_{1}^{\prime} p_{2}^{\prime} \ldots p_{k}^{\prime} p_{k+1}= \\
& \left(p_{k+1}^{\prime}-p_{k+1}\right) \sum_{i=0}^{k-1}(-1)^{k-i+1} S_{k}^{i}+p_{1}^{\prime} p_{2}^{\prime} \ldots p_{k}^{\prime} p_{k+1}= \\
& \left(p_{k+1}^{\prime}-p_{k+1}\right) p_{0}+p_{1}^{\prime} p_{2}^{\prime} \ldots p_{k}^{\prime} p_{k+1}=R(G),
\end{aligned}
$$

just as expected. Case of even $k$ is proved in similar way.

\section{Computational Algorithm and Case Studies}

Based on the presented results we propose the modification of the algorithm for strict calculation of the graph reliability that is discussed in 11. Briefly this modification looks as follows (we assume that a graph under consideration has connected structure).

Step 0 (preliminary). Remove all attached trees (if exist) from a graph, $\omega$ is the production of reliabilities of all removed edges.

Step 1. Substitute all simple chains (if exist) by edges according to formulas from [1], $\nu$ is the corresponding factor (if there are no simple chains in a graph then $\nu=1$ ).

Step 2. Decompose a graph into $k$ blocks (if decomposition is impossible, then $k=1$ and the only block is a graph itself) $G_{i} . R(G)=\nu \prod_{i=1}^{k} R\left(G_{i}\right)$. For each $G_{i}$ go to next step.(if possible)

Step 3. If graph is disconnected or allows direct computation then return 0 or calculated value, correspondingly. Otherwise go to next step.

Step 4. If there is a 2-node cut in a graph then use equations (2) or (9) for recursion. Otherwise go to next step.

Step 5. Choose an arbitrary edge $u$ with reliability $\varepsilon$ for recursive branching: $R(G)=\nu\left[\varepsilon R\left(G^{*}\right)+(1-\varepsilon) R(G \backslash e)\right]$. Here $G^{*}$ is a graph $G$ contracted by an edge $u$. Recursion starts from the Step 1 .

Step 6 (final). Calculated value is multiplied by $\omega$.

For case studies we use random graphs obtained according the algorithms from [5]. We have calculated the reliability of 100 random networks which structures that include 5 subgraphs corresponding to LANs and 5 additional edges for their interconnection. Each subgraph has 40 nodes and 60 edges thus each testing graph has 200 nodes and 305 edges. For experiments we have used the computer with the processor Intel Celeron $1000 \mathrm{MHz}, \mathrm{RAM}=320 \mathrm{Mb}$. Average time for a random graph is about $80 \mathrm{sec}$ while the previous algorithm that in [1] was 
proven to be fastest at that time gives no results in an hour. At the same time the proposed algorithm is slightly slower for complete graphs (for $K_{9}$ we have $47 \mathrm{sec}$ and $47.6 \mathrm{sec}$ correspondingly) as a result of unfruitful tests on the Step 3. Thus our new algorithm has higher efficiency for graphs with 2-node cuts initially or when a graph has low density and do 2-node cuts are obtained often enough during the branching process.

\section{Conclusion}

In this paper we have shown how to use 2-node cuts for speeding up the process of obtaining the exact reliability of networks with reliable nodes and unreliable edges. Together with previous technique based on use of simple chains it allows calculation of reliability of LANs in reasonable time. We think that our method can be used for topological optimization of networks using the method proposed in [6] in which the calculation of a graph reliability is one of the main subgoals. In the nearest future we will present results concerning case of multiple 2-node cuts and of cuts of higher capacity. Future researches can concern the exact calculation of reliability for networks with unreliable nodes also.

\section{Acknowledgment}

This research was supported by MIC, Korea under ITRC IITA-2005-(C10900501-0019) and RFBR.

\section{References}

1. Rodionova, O.K., Rodionov, A.S., and Choo, H.: Network Probabilistic Connectivity: Exact Calculation with Use of Chains. ICCSA-2004, Springer LNCS. 3046 (2004) 315-324

2. Moore, E.F., Shannon, C.E.: Reliable Circuits Using Less Reliable Relays. J. Franclin Inst. 262 (1956) 191-208

3. Shooman, A.M., Kershenbaum, A.: Exact Graph-Reduction Algorithms for Network Reliability Analysis. Proc. GLOBECOM' 91. 2 (1991) 1412-1420

4. Shooman, A.M.: Algorithms for Network Reliability and Connection Availability Analysis. Electro/95 Int. Professional Program Proc. (1995) 309-333

5. Rodionov, A.S., and Choo, H.: On Generating Random Network Structures: Connected Graphs. Proceedings of the International Conference on Information Networking ICOIN 2004, Vol. III (2004) 1145-1152

6. T. Koide, S. Shinmori and H. Ishii: Topological optimization with a network reliability constraint. Discrete Appl. Math., 115, Issues 1-3 (2001) 135-149 